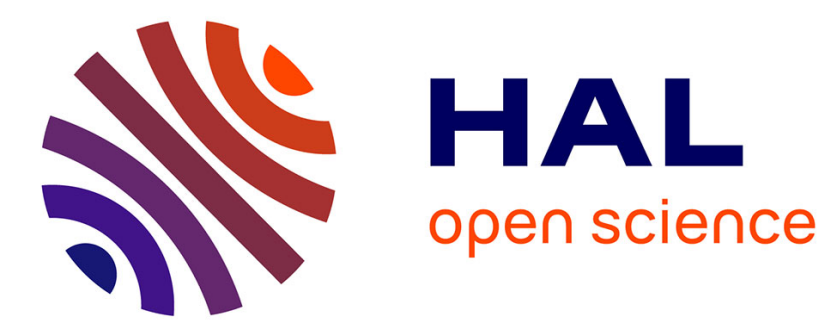

\title{
Study of the anisotropy of a passive scalar field at the level of dissipation
}

\author{
Michel Gonzalez
}

\section{To cite this version:}

Michel Gonzalez. Study of the anisotropy of a passive scalar field at the level of dissipation. Physics of Fluids, 2000, 12 (9), pp.2302 - 2310. 10.1063/1.1287516 . hal-01437151

\section{HAL Id: hal-01437151 \\ https://hal.science/hal-01437151}

Submitted on 17 Jan 2017

HAL is a multi-disciplinary open access archive for the deposit and dissemination of scientific research documents, whether they are published or not. The documents may come from teaching and research institutions in France or abroad, or from public or private research centers.
L'archive ouverte pluridisciplinaire HAL, est destinée au dépôt et à la diffusion de documents scientifiques de niveau recherche, publiés ou non, émanant des établissements d'enseignement et de recherche français ou étrangers, des laboratoires publics ou privés. 


\title{
Study of the anisotropy of a passive scalar field at the level of dissipation
}

\author{
Michel Gonzalez \\ CNRS UMR 6614/CORIA Université de Rouen, 76821 Mont-Saint-Aignan Cedex, France
}

(Received 4 January 2000; accepted 5 June 2000)

\begin{abstract}
The anisotropy of a passive scalar field at the level of second-order moments of the scalar derivatives is studied starting from the exact equations for the components of the second-rank tensor $\mathcal{E}$ defined by $\mathcal{E}_{i j}=2 D \overline{\left(\partial \theta^{\prime} / \partial x_{i}\right)\left(\partial \theta^{\prime} / \partial x_{j}\right)}$ ( $D$ is the molecular diffusivity of scalar $\theta$ ). Analysis requires also the equations for the components of a mixed tensor defined by the correlations between scalar and velocity gradients. After the examination of this set of equations, it is conjectured that, in the case of forcing by a mean scalar gradient in isotropic turbulence, the anisotropy of tensor $\mathcal{E}$ is produced by cliffs of temperature occurring in the direction of the mean gradient. A model for this mechanism is proposed including possible indirect influence of shear through the large-scale anisotropy of the scalar field. Predictions in the situation where a mean scalar gradient is combined with homogeneous shear agree tolerably well with experimental data. This suggests that the proposed picture describing production of small-scale anisotropy implied by mean gradient forcing has to be completed. (C) 2000 American Institute of Physics. [S1070-6631(00)50609-6]
\end{abstract}

\section{INTRODUCTION}

Investigating contamination of small scales by largescale features such as anisotropic forcing is akin to the efforts made for testing the concepts introduced in Kolmogorov's theory. In this respect, the passive scalar has become a subject of growing interest since local isotropy of scalar fields in shear flows has been seriously questioned. ${ }^{1,3-6}$ The relevance of this problem has furthermore been confirmed by experiments showing that, in isotropic turbulence, forcing by a mean scalar gradient results in a small-scale anisotropy of the scalar field which persists when Reynolds number is increased. ${ }^{7,8}$

A measure of the fine-scale anisotropy of the scalar field can be derived from various criteria among which are the value of the derivative skewness and refined tests such as checking to what extent the derivative spectra agree with their theoretical, isotropic expressions. ${ }^{9}$ As shown by Van Atta, ${ }^{9}$ anisotropy estimated through second-order moments of scalar derivatives is not inconsistent with isotropy at the smallest scales since departures from isotropy measured in this way may be explained by anisotropy at the low-wavenumber end of the gradient spectra. Possible contamination of the scalar field at this level, however, deserves investigation at least with regard to the question of the validity of isotropy arguments which are set forth in some scalar dissipation measurements and models.

The present work is focused on the anisotropy felt by a passive scalar field at the level of dissipation as a consequence of forcing by mean gradients. The set of exact equations for the components of tensors $\mathcal{E}$ and $\mathcal{F}$ defined, respectively, by $\mathcal{E}_{i j}=2 D \overline{\left(\partial \theta^{\prime} / \partial x_{i}\right)\left(\partial \theta^{\prime} / \partial x_{j}\right)}$ and $\mathcal{F}_{i j k}$ $=D \overline{\left(\partial \theta^{\prime} / \partial x_{i}\right)\left(\partial u_{k}^{\prime} / \partial x_{j}\right)}$ is considered with intent to bring out the influence of large-scale forcing. The task is delicate in that these equations include not only terms expressing direct action of forcing but also unclosed terms in the form of higher order correlations between gradients which are $a$ priori not free from anisotropic effects. Thereby, the study is based partly on the analysis of exact equations, partly on modeling. After the general equations are given (Sec. II), analysis in the case of forcing by a scalar gradient applied in isotropic turbulence is undertaken (Sec. III); a mechanism is put forward for explaining anisotropy of tensor $\mathcal{E}$ in this situation and a model is subsequently devised. An algebraic modeling of the components of $\mathcal{F}$ is also proposed (Sec. IV). Finally, the case of forcing by combined scalar gradient and shear is considered; comparisons of model predictions with experimental data are reported and discussed (Sec. V).

\section{GENERAL EQUATIONS}

\section{A. Scalar gradients correlations}

A second-rank tensor, $\mathcal{E}$, is defined as

$$
\mathcal{E}_{i j}=2 D \frac{\overline{\partial \theta^{\prime} \partial \theta^{\prime}}}{\partial x_{i} \partial x_{j}},
$$

where $D$ is the molecular diffusivity of the scalar quantity, $\theta$. The overbar denotes Reynolds averaging and prime fluctuating quantities. The components of $\mathcal{E}$ have the dimension of scalar variance dissipation; the mean dissipation rate of the scalar fluctuations energy, $\overline{\theta^{\prime 2}} / 2$, is nothing but the half trace of $\mathcal{E}: \epsilon_{\theta}=\mathcal{E}_{\alpha \alpha} / 2$. The equation for $\mathcal{E}_{i j}$ is derived from the instantaneous convection-diffusion equation for $\theta$ with the assumptions of incompressibility and constant diffusivity. The procedure is quite standard and is not reported here. Dropping transport terms (which is justified in the homogeneous situations which will be considered in the following) and in the limit of large Reynolds and Péclet numbers: 


$$
\begin{aligned}
\frac{d \mathcal{E}_{i j}}{d t}= & -\mathcal{E}_{i \alpha} \frac{\partial \bar{u}_{\alpha}}{\partial x_{j}}-\mathcal{E}_{j \alpha} \frac{\partial \bar{u}_{\alpha}}{\partial x_{i}}-2\left(\mathcal{F}_{i j \alpha}+\mathcal{F}_{j i \alpha}\right) \frac{\partial \bar{\theta}}{\partial x_{\alpha}}+\mathcal{S}_{i j} \\
& +\mathcal{D}_{i j} .
\end{aligned}
$$

In Eq. (1), $u_{i}$ is the $i$ th component of the velocity vector, $\mathcal{F}_{i j k}$ are the components of a mixed tensor:

$$
\mathcal{F}_{i j k}=D \overline{\frac{\partial \theta^{\prime}}{\partial x_{i}} \frac{\partial u_{k}^{\prime}}{\partial x_{j}},}
$$

and

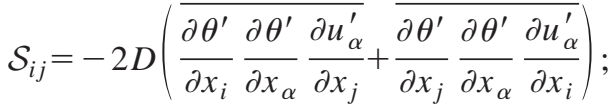

$$
\begin{aligned}
& \mathcal{D}_{i j}=-4 D^{2} \frac{\partial}{\partial x_{\alpha}}\left(\frac{\partial \theta^{\prime}}{\partial x_{i}}\right) \frac{\partial}{\partial x_{\alpha}}\left(\frac{\partial \theta^{\prime}}{\partial x_{j}}\right) .
\end{aligned}
$$

The terms of Eq. (1) can be interpreted as follows. The first three terms on right-hand side (rhs) are production terms and can be viewed as representing large-scale forcing by mean velocity and scalar gradients. The fourth and fifth terms represent, respectively, production by stretching and destruction by molecular dissipation. The order-ofmagnitude analysis proves that although the stretching and dissipation terms are of order $\operatorname{Re}_{\lambda}$ larger than production terms $\left(\operatorname{Re}_{\lambda}\right.$ being the Reynolds number based on the Taylor microscale, $\lambda)$, their difference is of the same order as the latter. $^{10,11}$

An outstanding feature of the $\mathcal{E}_{i j}$ equation is the lack of terms to which a return to isotropy can explicitly be ascribed. This sharply contrasts with the case of the Reynolds stresses dissipation tensor, $\epsilon_{i j}=2 \nu \overline{\left(\partial u_{i}^{\prime} / \partial x_{\alpha}\right)\left(\partial u_{j}^{\prime} / \partial x_{\alpha}\right)}$ ( $\nu$ is the kinematic viscosity) the equation of which includes a pressure term ensuring redistribution. ${ }^{12-14}$ However, this does not mean that the components of $\mathcal{E}$ do not experience any return to isotropy. Indeed, if such a mechanism were not present then, experiments would reveal ever-increasing anisotropy of $\mathcal{E}$, which is not the case. Now, examination of Eq. (1) suggests that return to isotropy has to be ascribed to stretching and dissipation since other terms represent anisotropic forcing. Molecular dissipation appears, moreover, to be the best candidate. In this respect, note that Lumley ${ }^{15}$ has put forward the deviatoric part of viscous dissipation acts, in addition to the pressure term, as a redistribution mechanism in the equation for the Reynolds stresses.

\section{B. Scalar gradients-velocity gradients correlations}

It is worth noting, from Eq. (1), that forcing by mean shear involves the components of tensor $\mathcal{E}$ itself whereas forcing by mean scalar gradients set the mixed tensor, $\mathcal{F}$, into play. Derivation of an evolution equation for the components $\mathcal{F}_{i j k}$ is similar to that of the $\mathcal{E}_{i j}$ equation. The only difference, this time, lies in the use of the Navier-Stokes equation in addition to the convection-diffusion equation for $\theta$. With the same previous assumptions (no transport terms, large Reynolds, and Péclet numbers), this equation is written as follows:

$$
\begin{aligned}
\frac{d \mathcal{F}_{i j k}}{d t}= & -\mathcal{F}_{i j \alpha} \frac{\partial \bar{u}_{k}}{\partial x_{\alpha}}-\frac{1}{2} \mathrm{Sc}^{-1} e_{i j \alpha k} \frac{\partial \bar{\theta}}{\partial x_{\alpha}}-\mathcal{F}_{\alpha j k} \frac{\partial \bar{u}_{\alpha}}{\partial x_{i}} \\
& -\mathcal{F}_{i \alpha k} \frac{\partial \bar{u}_{\alpha}}{\partial x_{j}}+\Sigma_{i j k}+\Delta_{i j k}+\Pi_{i j k} .
\end{aligned}
$$

$\mathrm{Sc}$ is the Schmidt number, $\mathrm{Sc}=\nu / D, \rho_{0}$, the density, and $\left.e_{i j k l}=2 \nu \overline{\left(\partial u_{k}^{\prime} / \partial x_{i}\right)\left(\partial u_{l}^{\prime} / \partial x_{j}\right.}\right)$. In addition,

$$
\begin{aligned}
& \Sigma_{i j k}=-D\left(\overline{\frac{\partial \theta^{\prime}}{\partial x_{\alpha}} \frac{\partial u_{k}^{\prime}}{\partial x_{j}} \frac{\partial u_{\alpha}^{\prime}}{\partial x_{i}}}+\overline{\frac{\partial \theta^{\prime}}{\partial x_{i}} \frac{\partial u_{k}^{\prime}}{\partial x_{\alpha}} \frac{\partial u_{\alpha}^{\prime}}{\partial x_{j}}}\right), \\
& \Delta_{i j k}=-2 D^{2} \frac{\partial}{\partial x_{\alpha}}\left(\frac{\partial \theta^{\prime}}{\partial x_{i}}\right) \frac{\partial}{\partial x_{\alpha}}\left(\frac{\partial u_{k}^{\prime}}{\partial x_{j}}\right) \\
& -D^{2}(1-\mathrm{Sc}) \overline{\frac{\partial \theta^{\prime}}{\partial x_{i}} \frac{\partial^{2}}{\partial x_{\alpha} \partial x_{\alpha}}\left(\frac{\partial u_{k}^{\prime}}{\partial x_{j}}\right)}, \\
& \Pi_{i j k}=-\frac{D}{\rho_{0}} \overline{\frac{\partial \theta^{\prime}}{\partial x_{i}} \frac{\partial^{2} p^{\prime}}{\partial x_{j} \partial x_{k}}} .
\end{aligned}
$$

The terms of Eq. (2) can be interpreted, again, as production by mean gradients (the first to fourth term on the rhs), production by stretching and destruction by molecular dissipation (respectively, the fifth and sixth terms). In addition, Eq. (2) includes a pressure term (the last term on the rhs) arising from the interaction between the scalar and the velocity fields in the form of a scalar gradient-pressure Hessian correlation. The order-of-magnitude analysis of Eq. (2) can be undertaken in the same way as for Eq. (1).

Interestingly, production of $\mathcal{F}_{i j k}$ by mean scalar gradients (the second term on the rhs) involves the components of the fourth-rank tensor, $e_{i j k l}$. This implies that forcing by a mean scalar gradient affects scalar dissipation through the interaction between the large scales of the scalar field and the dissipative scales of the velocity field. Such a mechanism could thereby be a cause of contamination of the scalar dissipation field by the anisotropy (if any) of the turbulence energy dissipation. The corresponding term is, however, Schmidt number dependent as it is proportional to $\mathrm{Sc}^{-1}$. The previous interaction is thus ineffective at large Schmidt number but is enhanced if the Schmidt number assumes small or moderate values.

The pressure term, $\Pi_{i j k}$, raises a special problem for it includes the pressure Hessian which plays an important role in vortex dynamics. ${ }^{16,17}$ The difficulty in modeling the pressure Hessian lies in its nonlocal nature. As shown by Ohkitani and Kishiba, ${ }^{16}$ it can be expressed as

$$
\frac{\partial^{2} p}{\partial x_{i} \partial x_{j}}=\frac{1}{3} \frac{\partial^{2} p}{\partial x_{\alpha} \partial x_{\alpha}} \delta_{i j}+K_{i j},
$$

in which $K_{i j}$ is a nonlocal term in the form of an integral over space. The restricted Euler model proposed by Vieillefosse ${ }^{18}$ keeps only the isotropic, local part of the pressure Hessian. It has been $\operatorname{shown}^{19}$ that this approximation retains the main features of the velocity gradients behavior, at least in isotropic or homogeneous sheared turbulence. Martín et al. ${ }^{20}$ recently devised a model for the evolution of the velocity gradients neglecting the anisotropic part of the 
pressure Hessian and retrieved most of the known properties of the small scales of turbulence. Restricting the pressure Hessian to its isotropic part implies, for the pressure term of the $\mathcal{F}_{i j k}$ equation,

$$
\Pi_{i j k}=-\frac{D}{3 \rho_{0}} \frac{\overline{\partial \theta^{\prime}}}{\partial x_{i}} \frac{\partial^{2} p^{\prime}}{\partial x_{\alpha} \partial x_{\alpha}} \delta_{j k} .
$$

In an incompressible flow, the pressure Laplacian is easily derived from the equation for the fluctuating velocity which finally leads to

$$
\Pi_{i j k}=\frac{1}{3}\left(D \overline{\frac{\partial \theta^{\prime}}{\partial x_{i}} \frac{\partial u_{\alpha}^{\prime}}{\partial x_{\beta}} \frac{\partial u_{\beta}^{\prime}}{\partial x_{\alpha}}}+2 \mathcal{F}_{i \beta \alpha} \frac{\partial \bar{u}_{\beta}}{\partial x_{\alpha}}\right) \delta_{j k} .
$$

\section{FORCING BY A MEAN SCALAR GRADIENT IN ISOTROPIC TURBULENCE}

\section{A. Analysis}

The effect of forcing by a scalar gradient can be studied in the basic configuration of the uniform, transverse, mean scalar gradient in isotropic turbulence as first suggested by Corrsin. $^{21}$ Further experiments in decaying grid turbulence ${ }^{22-24}$ have confirmed the steadiness of the scalar gradient with downstream distance as well as the fact that reasonable cross-stream homogeneity of the scalar field can be ensured provided that the scalar profile is generated by a device independent of the grid.

In the following, it is assumed that a cross-stream, passive, scalar gradient $\Gamma$ is applied in isotropic turbulence in such a way that

$$
\bar{\theta}=\Gamma x_{2}, \quad \Gamma=\text { constant }, \quad \Gamma>0 .
$$

Assuming unity Schmidt number, Eqs. (1) and (2) can then be written as

$$
\begin{aligned}
& \frac{d \mathcal{E}_{11}}{d t}=-4 \Gamma \mathcal{F}_{112}+\mathcal{S}_{11}+\mathcal{D}_{11}, \\
& \frac{d \mathcal{E}_{22}}{d t}=-4 \Gamma \mathcal{F}_{222}+\mathcal{S}_{22}+\mathcal{D}_{22}, \\
& \frac{d \mathcal{E}_{33}}{d t}=-4 \Gamma \mathcal{F}_{332}+\mathcal{S}_{33}+\mathcal{D}_{33}, \\
& \frac{d \mathcal{F}_{112}}{d t}=-\frac{1}{2} \Gamma e_{1122}+\Sigma_{112}+\Delta_{112}, \\
& \frac{d \mathcal{F}_{222}}{d t}=-\frac{1}{2} \Gamma e_{2222}+\Sigma_{222}+\Delta_{222}+\Pi_{222}, \\
& \frac{d \mathcal{F}_{332}}{d t}=-\frac{1}{2} \Gamma e_{3322}+\Sigma_{332}+\Delta_{332} .
\end{aligned}
$$

The pressure term, $\Pi_{222}$, is approximated as shown in Sec. II [Eq. (3)] and, in the present case of zero mean shear, reduces to

$$
\Pi_{222}=\frac{D}{3} \overline{\frac{\partial \theta^{\prime}}{\partial x_{2}} \frac{\partial u_{\alpha}^{\prime}}{\partial x_{\beta}} \frac{\partial u_{\beta}^{\prime}}{\partial x_{\alpha}} .}
$$

The anisotropy of tensor $\mathcal{E}$ is described by Eqs. (4)-(9). Isotropy of the velocity field (which implies $e_{i j 22}=0$ and hence $\mathcal{F}_{i j 2}=0$ when $i \neq j$ ) precludes off-diagonal components. It is worth noticing that the mean scalar gradient affects all three diagonal components of $\mathcal{E}$ through interaction with tensor $\mathcal{F}$ [Eqs. (4)-(6)]. In passing, components $\mathcal{F}_{112}$ and $\mathcal{F}_{332}$ which play, respectively, on $\mathcal{E}_{11}$ and $\mathcal{E}_{33}$ are promoted in regions of the flow where vorticity has one component normal to the mean scalar gradient, whereas $\mathcal{F}_{222}$ (which plays on $\mathcal{E}_{22}$ ) is promoted in regions where strain acts along the mean gradient.

Although all three diagonal components of $\mathcal{E}$ are influenced by large-scale forcing, it is experimentally proved that, in the present case, $\mathcal{E}_{22} / \mathcal{E}_{11}$ lies between 1.2 and 1.6 (and that $\mathcal{E}_{11} \simeq \mathcal{E}_{33}$ ) independently of Reynolds number. ${ }^{7,8}$ Starting from an isotropic situation, the predominance of $\mathcal{E}_{22}$ can be explained only by some mechanism promoting this component. However, direct production of $\mathcal{E}_{22}$ occurs through $\mathcal{F}_{222}$ which, itself, is produced by $-e_{2222} \Gamma / 2$ (note that, here, $\mathcal{F}_{112}, \mathcal{F}_{222}$, and $\mathcal{F}_{332}$ are negative). Now, in isotropic turbulence, this latter term is only half the production terms of $\mathcal{F}_{112}$ and $\mathcal{F}_{332}$ since $e_{2222}=e_{1122} / 2=e_{3322} / 2$. In addition, it will be shown in the following that, in those strain regions where $\mathcal{F}_{222}$ is promoted, the pressure term $\Pi_{222}$ is likely to act as a destruction mechanism. Hence, predominance of component $\mathcal{E}_{22}$ over $\mathcal{E}_{11}$ and $\mathcal{E}_{33}$ cannot be understood by direct production. Most probably, values of $\mathcal{E}_{22} / \mathcal{E}_{11}$ larger than unity have to be explained through an influence of the mean gradient on stretching and dissipation of $\mathcal{F}_{i j k}$ resulting in $\Sigma_{222}+\Delta_{222}<\Sigma_{112}+\Delta_{112}$, possibly implying $\mathcal{F}_{222}<\mathcal{F}_{112}$ and/or a similar effect felt by the stretching and dissipation of $\mathcal{E}_{i j}$, both of these mechanisms leading to enhancement of $\mathcal{E}_{22}$ with respect to $\mathcal{E}_{11}$ and $\mathcal{E}_{33}$.

\section{B. Proposed mechanism for the anisotropy of $\mathcal{E}$}

There is now ample evidence of a persistent skewness of the scalar derivative $\partial \theta / \partial x_{2}$ of the same sign as the one of the imposed mean gradient ${ }^{7,8,23,25-27}$ (positive in the case under study). Besides, recent experiments and simulations ${ }^{7,26,27}$ have confirmed that, even in unsheared situations, forcing by a mean scalar gradient induces ramp-cliff type events in the scalar time signal. The latter have been invoked to explain the skewness of $\partial \theta / \partial x_{2}$ and can be understood as resulting from the transit, along the gradient, of fluid lumps moving from the low- $\theta$ region toward the high- $\theta$ region and vice versa (Fig. 1). ${ }^{23,25}$ Jumps in $\partial \theta / \partial x_{2}$ are connected with the upstream front of these fluid structures. If, following for instance Budwig et al., ${ }^{23}$ the existence of a local stagnation region upstream of each lump is assumed then jumps in $\partial \theta / \partial x_{2}$ are strongly correlated with negative jumps in $\partial u_{2} / \partial x_{2}$.

An inference regarding the pressure term $\Pi_{222}$ can be drawn from the above-mentioned picture. This term can be written in function of vorticity $\omega$ and strain $\sigma$ as

$$
\Pi_{222}=-\frac{D}{3} \overline{\frac{\partial \theta^{\prime}}{\partial x_{2}}\left(\frac{1}{2} \omega^{2}-\sigma^{2}\right)},
$$

with 

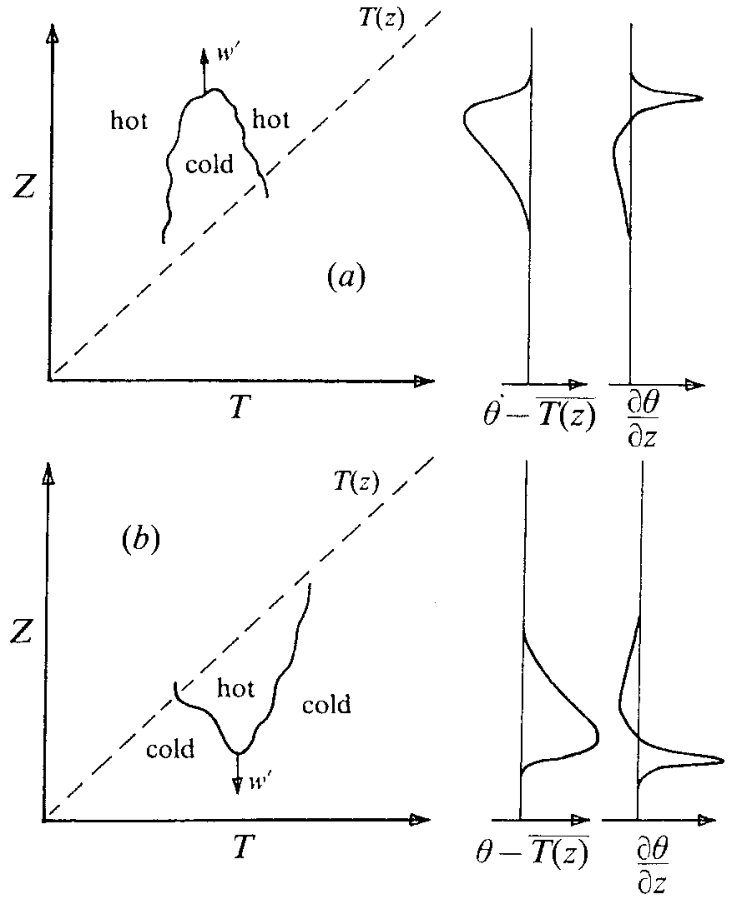

FIG. 1. Phenomenological model of fluid lumps explaining ramp-cliff events in the scalar signal and connected jumps in the instantaneous scalar derivative under influence of a mean scalar gradient [taken from Thoroddsen and Van Atta (1992) (Ref. 25) in which the scalar is temperature].

$$
\begin{aligned}
\sigma^{2} & =\frac{1}{2}\left(\frac{\partial u_{\alpha}^{\prime}}{\partial x_{\beta}} \frac{\partial u_{\alpha}^{\prime}}{\partial x_{\beta}}+\frac{\partial u_{\alpha}^{\prime}}{\partial x_{\beta}} \frac{\partial u_{\beta}^{\prime}}{\partial x_{\alpha}}\right), \\
\omega^{2} & =\frac{\partial u_{\alpha}^{\prime}}{\partial x_{\beta}} \frac{\partial u_{\alpha}^{\prime}}{\partial x_{\beta}}-\frac{\partial u_{\alpha}^{\prime}}{\partial x_{\beta}} \frac{\partial u_{\beta}^{\prime}}{\partial x_{\alpha}} .
\end{aligned}
$$

It follows that $\Pi_{222}$ is weak in shear regions (where $\omega^{2} / 2$ $\simeq \sigma^{2}$ ) and does not affect $\mathcal{F}_{222}$ in the latter. Furthermore, in strain regions, where $\mathcal{F}_{222}$ is mainly produced, $\Pi_{222}$ $\simeq D / 3 \overline{\left(\partial \theta^{\prime} / \partial x_{2}\right) \sigma^{2}}$, that is, $\Pi_{222}>0$, which implies, as previously mentioned, that $\Pi_{222}$ acts on $\mathcal{F}_{222}$ as a destruction mechanism. The positivity of $\Pi_{222}$ is reinforced by the term $\overline{\partial \theta^{\prime} / \partial x_{2}\left(\partial u_{2}^{\prime} / \partial x_{2}\right)^{2}}$ as a result of the correlation of jumps in $\partial \theta / \partial x_{2}$ (positive in this situation) with those in $\partial u_{2} / \partial x_{2}$.

Another consequence of the previous phenomenological model is the enhancement of the stretching of $\mathcal{E}_{22}$ via the term $-D \overline{\left(\partial \theta^{\prime} / \partial x_{2}\right)^{2} \partial u_{2}^{\prime} / \partial x_{2}}$. The correlation of negative jumps in $\partial u_{2} / \partial x_{2}$ with jumps in $\partial \theta / \partial x_{2}$ caused by the fronts of the fluid lumps moving along $x_{2}$ direction indeed implies a positive contribution to this strain term. The order of magnitude of such a contribution can be estimated as follows. Taking for granted that the typical size of the fluid lumps is the integral scale, $\Lambda$, and that the thickness of their front is of the order of the Taylor microscale, $\lambda$, the magnitude of a jump in the scalar derivative is $\Gamma \Lambda / \lambda .^{7}$ The resulting contribution to the stretching of $\mathcal{E}_{22}$ is therefore of order

$$
D \frac{u_{2}^{\prime}}{\lambda}\left(\Gamma \frac{\Lambda}{\lambda}\right)^{2} \sim D \frac{u_{2}^{\prime}}{\lambda} \frac{\theta^{\prime 2}}{\lambda^{2}}
$$

and is larger by $\operatorname{Re}_{\lambda}$ than the production term in the $\mathcal{E}_{22}$ equation (which scales as $D u_{2}^{\prime} \theta^{\prime 2} / \lambda^{2} \Lambda$ ). However, the above-mentioned contribution is to be weighted by the fraction of ramp-cliff events which Tong and Warhaft, ${ }^{7}$ from their measurements of the probability density function of $\partial \theta / \partial x_{2}$, show to vary approximately as $\operatorname{Re}_{\lambda}^{-1}$. On the whole, the effect of cliff events on the stretching of $\mathcal{E}_{22}$ thereby appears to be only slightly Reynolds number dependent. Nevertheless, this contribution cannot be neglected in the budget of $\mathcal{E}_{22}$ since it is of the same order as other terms (including the sum of stretching and dissipation). It is likely that, via the same mechanism, the component $\mathcal{F}_{222}$ is reinforced as well.

Finally, the above-mentioned scenario can be summarized in a different, although equivalent, way. Isotropy of the velocity field precludes any preferential direction of strain. However, the compressive strain events occurring along the mean scalar gradient $\partial \bar{\theta} / \partial x_{2}$ (corresponding, for instance, to the stagnation region upstream of the fronts moving in $x_{2}$ direction) make the small scales of the scalar feel the largescale asymmetry imposed by the mean gradient. This results, here, in enhancement of $\mathcal{E}_{22}$ with respect to $\mathcal{E}_{11}$ and $\mathcal{E}_{33}$. In the following, a model for this mechanism is proposed.

\section{Consequence for modeling}

Accounting for anisotropy at the level of stretching and dissipation of $\mathcal{E}$ implies that the components of the latter can be written as

$$
\mathcal{S}_{i j}=\mathcal{S}_{i j}^{0}+\mathcal{S}_{i j}^{A}, \quad \mathcal{D}_{i j}=\mathcal{D}_{i j}^{0}+\mathcal{D}_{i j}^{A} .
$$

$\mathcal{S}_{i j}^{0}$ and $\mathcal{D}_{i j}^{0}$ represent the isotropic parts of $\mathcal{S}_{i j}$ and $\mathcal{D}_{i j} \cdot \mathcal{S}_{i j}^{A}$ and $\mathcal{D}_{i j}^{A}$ are the components of the anisotropic parts, represented by traceless tensors $\left(\mathcal{S}_{\alpha \alpha}^{A}=0, \mathcal{D}_{\alpha \alpha}^{A}=0\right)$.

As already mentioned, although terms $\mathcal{S}_{i j}^{0}$ and $\mathcal{D}_{i j}^{0}$ are of order $\operatorname{Re}_{\lambda}$ when compared to other terms, their sum is of order $\operatorname{Re}_{\lambda}^{0}$. In the absence of forcing, Newman et al. ${ }^{28}$ model $\mathcal{S}_{\alpha \alpha}^{0}+\mathcal{D}_{\alpha \alpha}^{0}$ as a dissipation mechanism (of order $\operatorname{Re}_{\lambda}^{0}$ ):

$$
\mathcal{S}_{\alpha \alpha}^{0}+\mathcal{D}_{\alpha \alpha}^{0} \equiv 2 \mathcal{N}^{0}=-2 \psi_{\theta} \frac{\epsilon_{\theta}^{2}}{\overline{\theta^{\prime 2}}},
$$

where $\psi_{\theta}$, which is a functional of tensorial invariants, is restricted to a linear function of $R$, the scalar-to-velocity time scale ratio $\left(R=\overline{\theta^{\prime 2}} \epsilon / q^{2} \epsilon_{\theta}\right.$, with $q^{2} / 2$ and $\epsilon$ being, respectively, the kinetic energy of turbulence and its dissipation rate):

$$
\psi_{\theta}=-\left(C_{S} R+C_{D}\right) .
$$

Constants $C_{S}$ and $C_{D}$ have already been determined. ${ }^{28-32}$ Lumley ${ }^{15}$ previously suggested that, in anisotropic situations, the decay of $\epsilon_{\theta}$ should depend on the anisotropy of the scalar field. Following this idea, Zeman and Lumley ${ }^{32}$ include a production term stemming from the anisotropy of the scalar field in their modeled $\epsilon_{\theta}$ equation. Generalizing this concept to the components $\mathcal{S}_{i j}^{0}+\mathcal{D}_{i j}^{0}$ leads to

$$
\mathcal{S}_{i j}^{0}+\mathcal{D}_{i j}^{0} \equiv \frac{2}{3}\left(\mathcal{N}^{0}+\mathcal{N}^{A}\right) \delta_{i j}
$$


where $\mathcal{N}^{A}$ is a term resulting from large-scale anisotropy which, here, is written as

$$
\mathcal{N}^{A}=-C_{A} \overline{u_{\alpha}^{\prime} \theta^{\prime}} \frac{\partial \bar{\theta}}{\partial x_{\alpha}} \frac{\epsilon_{\theta}}{\overline{\theta^{\prime 2}}} .
$$

$C_{A}$ is a constant which will be estimated later on.

Now, the model for $\mathcal{S}_{i j}+\mathcal{D}_{i j}$ has to include the effect of large-scale anisotropy on $\mathcal{S}_{i j}$ due to mean scalar gradient. Following the discussion of Sec. II B, it is proposed to model this mechanism by a term of order $\mathrm{Re}_{\lambda}^{0}$ representing production of $\mathcal{E}_{i j}$ as a result of the transit of fluid lumps along the mean gradient. In this respect, a general expression could be

$$
\mathcal{P}_{i j}^{A}=-2 C_{A} \overline{u_{\alpha}^{\prime} \theta^{\prime}} \frac{\partial \bar{\theta}}{\partial x_{\alpha}}\left(\frac{b_{i} b_{j}}{b_{\beta} b_{\beta}}-\frac{1}{3} \delta_{i j}\right) \frac{\epsilon_{\theta}}{\overline{\theta^{\prime 2}}}
$$

with

$$
b_{i}=\frac{\overline{u_{i}^{\prime} \theta^{\prime}}}{\left(q^{2} \overline{\theta^{\prime 2}}\right)^{1 / 2}} .
$$

The nonlinear term $b_{i} b_{j} / b_{\beta} b_{\beta}$ represents large-scale anisotropy of the scalar field and is introduced for including indirect effect of shear. The term in $\delta_{i j} / 3$ ensures that $\mathcal{P}_{\alpha \alpha}^{A}=0$. In the modeled expression for $\mathcal{S}_{i j}+\mathcal{D}_{i j}$, it will cancel with $2 \mathcal{N}^{A} \delta_{i j} / 3$.

It is to be stressed that there is apparently neither theoretical nor experimental information regarding the return to isotropy of the scalar field at the level of dissipation. At this stage, the only term of the $\mathcal{E}_{i j}$ equation to which such a mechanism could be ascribed is the anisotropic part of the dissipative term $\mathcal{D}_{i j}$, as already discussed in Sec. II A. In passing, note that the ramp-cliff events also cause jumps in the second-order spatial derivative along the mean scalar gradient, which enhances dissipation and most likely tends to balance their above-mentioned production effect. Recent experimental results ${ }^{7,8}$ show that anisotropy at the smallest scales of the scalar field does not seem to relax with increasing Reynolds number suggesting that return to isotropy could be represented by a term which does not depend on the latter. Using a relaxation-type expression, return to isotropy could be modeled as

$$
\mathcal{R}_{i j}^{A}=-\left(\mathcal{E}_{i j}-\frac{2}{3} \epsilon_{\theta} \delta_{i j}\right) \tau_{\mathcal{R}}^{-1} .
$$

In the above-mentioned expression, $\tau_{\mathcal{R}}$ is a characteristic time scale. In the absence of firm knowledge of this time scale and following the statement that return to isotropy is ensured by molecular dissipation, an obvious model consists in assuming that $\tau_{\mathcal{R}}^{-1}$ is proportional to the scalar frequency:

$$
\tau_{\mathcal{R}}^{-1}=\alpha_{\mathcal{R}} \frac{\epsilon_{\theta}}{\overline{\theta^{\prime 2}}} .
$$

An upper bound of $\alpha_{\mathcal{R}}$ can be estimated by noticing that some experimental and numerical results, $6,33,34$ display a stronger anisotropy for scalar gradients than for velocity gradients. This, indeed, leads one to surmise that return to isotropy is slower for the scalar gradients. Now, in the model of
Speziale and Gatski ${ }^{14}$ for the anisotropy of Reynolds stresses dissipation, the return-to-isotropy frequency is approximately $12 \epsilon / q^{2}$. It is therefore postulated that $\alpha_{\mathcal{R}}<12$. Furthermore, the relaxation frequency has to be larger than the dissipation one to ensure that, in the decaying case, return to isotropy effectively acts before complete small-scale mixing is achieved. With $C_{S} \simeq C_{D} \simeq 2$ (Refs. 28-32) and $R$ of order one, this implies $\alpha_{\mathcal{R}}>4$. In Sec. V, model predictions in the situation of the Tavoularis and Corrsin's experiment ${ }^{2,3}$ will be obtained with $\alpha_{\mathcal{R}}=7$.

The model for stretching and dissipation can finally be written in the following form:

$$
\mathcal{S}_{i j}+\mathcal{D}_{i j} \equiv \frac{2}{3}\left(\mathcal{N}^{0}+\mathcal{N}^{A}\right) \delta_{i j}+\mathcal{P}_{i j}^{A}+\mathcal{R}_{i j}^{A},
$$

and thus

$$
\begin{aligned}
\mathcal{S}_{i j}+\mathcal{D}_{i j} \equiv & -\frac{2}{3}\left(C_{S} R+C_{D}\right) \frac{\epsilon_{\theta}^{2}}{\overline{\theta^{\prime 2}}} \delta_{i j} \\
& -2 C_{A} \overline{u_{\alpha}^{\prime} \theta^{\prime}} \frac{\partial \bar{\theta}}{\partial x_{\alpha}} \frac{b_{i} b_{j}}{b_{\beta} b_{\beta}} \frac{\epsilon_{\theta}}{\overline{\theta^{\prime 2}}} \\
& -\alpha_{\mathcal{R}}\left(\mathcal{E}_{i j}-\frac{2}{3} \epsilon_{\theta} \delta_{i j}\right) \frac{\epsilon_{\theta}}{\overline{\theta^{\prime 2}}}
\end{aligned}
$$

In the present case, only $\mathcal{E}_{22}$ is affected through the production term $-2 C_{A} \overline{u_{2}^{\prime} \theta^{\prime}} \Gamma \epsilon_{\theta} / \overline{\theta^{\prime 2}}\left(b_{1}=b_{3}=0\right)$. If a mean shear (say, $\partial \bar{u}_{1} / \partial x_{2}$ ) were superposed upon the mean scalar gradient, then, since $b_{1}$ would be nonzero, the nonlinear term $b_{i} b_{j} / b_{\beta} b_{\beta}$ would make $\mathcal{E}_{11}$ feel, as well, the effect of cliffs.

Finally, using the above-mentioned model for $\mathcal{S}_{i j}+\mathcal{D}_{i j}$ in Eq. (1) and contracting the latter results in the following $\epsilon_{\theta}$ equation:

$$
\begin{aligned}
\frac{d \epsilon_{\theta}}{d t}= & -\mathcal{E}_{\alpha \beta} \frac{\partial \bar{u}_{\alpha}}{\partial x_{\beta}}-2 \mathcal{F}_{\alpha \alpha \beta} \frac{\overline{\partial \theta}}{\partial x_{\beta}} \\
& -C_{A} \overline{u_{\alpha}^{\prime} \theta^{\prime}} \frac{\partial \bar{\theta}}{\partial x_{\alpha}} \frac{\epsilon_{\theta}}{\overline{\theta^{\prime 2}}}-\left(C_{S} R+C_{D}\right) \frac{\epsilon_{\theta}^{2}}{\overline{\theta^{\prime 2}}} .
\end{aligned}
$$

The third term on the rhs, although under the form of production by mean scalar gradient, has a different origin than the second one since it stems from the modeling of the anisotropy of stretching. It can be compared, in fact, with the additional term that Zeman and Lumley ${ }^{32}$ have introduced in their $\epsilon_{\theta}$ model as a measure of anisotropy of the scalar field (but have written as $\overline{u_{\alpha}^{\prime} \theta^{\prime}} \overline{u_{\alpha}^{\prime} \theta^{\prime}}$ ). Current models include only one term representing production by mean scalar gradients; this is equivalent to model production due to interaction between $\mathcal{F}$ and the scalar gradient and production resulting from anisotropy at the level of stretching as a whole. This fact is used in Sec. IV for connecting the constants of the present model to the production constant of usual $\epsilon_{\theta}$ models [Eq. (14)]. 


\section{SCALAR GRADIENTS-VELOCITY GRADIENTS CORRELATIONS}

\section{A. Algebraic modeling}

Complete description of the anisotropy of tensor $\mathcal{E}$ requires modeling the $\mathcal{F}_{i j k}$ components. Note that models for the molecular dissipation of the scalar fluxes $\overline{u_{k}^{\prime} \theta^{\prime}}$ that is, the sum $(1+\mathrm{Sc}) \mathcal{F}_{\alpha \alpha k}$, have recently been proposed. ${ }^{35-37}$ In the present case, knowledge of the individual components of $\mathcal{F}$ is needed and the model has to be more general. If, instead of modeling the $\mathcal{F}_{i j k}$ equation, an algebraic model is devised, then, a natural way consists in writing $\mathcal{F}$ in function of the anisotropy of the scalar field. Nondimensional components of $\mathcal{F}$ defined as

$$
\mathcal{F}_{i j k}^{*}=\mathcal{F}_{i j k}\left(\epsilon \epsilon_{\theta}\right)^{-1 / 2},
$$

are written as follows:

$$
\mathcal{F}_{i j k}^{*}=\alpha_{1}\left(\delta_{i j}-\frac{b_{i} b_{j}}{b_{\alpha} b_{\alpha}}\right) b_{k}+\alpha_{3}\left(-3 \delta_{i k} b_{j}+\delta_{j k} b_{i}\right)
$$

This is tantamount to connect anisotropy at the level of dissipation with large-scale anisotropy.

Equation (12) ensures that $\mathcal{F}_{\alpha \alpha k}^{*} \propto b_{k}$ and that, in isotropic conditions, $\mathcal{F}_{i j k}^{*}=0$. Continuity is also satisfied since $\mathcal{F}_{i \alpha \alpha}^{*}=0$.

In passing, note that in the case of forcing by a mean scalar gradient $\Gamma\left(\bar{\theta}=\Gamma x_{2}\right)$ in isotropic turbulence: $\mathcal{F}_{112}^{*}$ $=\mathcal{F}_{332}^{*}=\alpha_{1} b_{2}$ and $\mathcal{F}_{222}^{*}=-2 \alpha_{3} b_{2}$. This obviously implies $\alpha_{1}>0$ and $\alpha_{3}<0$.

\section{B. The problem of unknown parameters}

At this stage, $\alpha_{1}, \alpha_{3}$, and $C_{A}$ are still to be determined. Experimental data on the $\mathcal{F}_{i j k}$ components would help in determining $\alpha_{1}$ and $\alpha_{3}$ but these are unfortunately rather scarce. ${ }^{2,11}$ First, it is attempted to get an estimate of $\alpha_{1}$ considering the case of forcing by a transverse mean scalar gradient in isotropic turbulence. Then, the measurements of Antonia and Browne ${ }^{11}$ in a plane jet are used for approximating the ratio $\alpha_{3} / \alpha_{1}$.

Using the model given by Eq. (10) in the equations for $\mathcal{E}_{11}$ and $\mathcal{E}_{22}$ as well as Eqs. (11) and (12) for expressing $\mathcal{F}_{112}$ and $\mathcal{F}_{222}$, the following equation is derived:

$$
\begin{aligned}
R_{\mathrm{eq}}^{1 / 2}\left(\alpha_{1}+2 \alpha_{3} \frac{\mathcal{E}_{11}}{\mathcal{E}_{22}}\right)= & \frac{C_{A}}{2}\left(\frac{\mathcal{E}_{11}}{\mathcal{E}_{22}}\right)-\frac{\alpha_{\mathcal{R}}-\left(C_{S} R_{\mathrm{eq}}+C_{D}\right)}{6\left(\mathcal{P}_{\theta} / \epsilon_{\theta}\right)_{\mathrm{eq}}} \\
& \times\left(1-\frac{\mathcal{E}_{11}}{\mathcal{E}_{22}}\right),
\end{aligned}
$$

where $\mathcal{E}_{11} / \mathcal{E}_{22}$ is the ratio of $\mathcal{E}_{11}$ to $\mathcal{E}_{22}$ at equilibrium. $\mathcal{P}_{\theta} / \epsilon_{\theta}$ is the production/dissipation ratio of the scalar variance $\left(\mathcal{P}_{\theta} / \epsilon_{\theta}=-\overline{u_{2}^{\prime} \theta^{\prime}} \Gamma / \epsilon_{\theta}\right)$, which, in the case of forcing by a uniform, transverse, mean scalar gradient, has experimentally been proved to tend to a constant value, as well as the scalar-to-velocity time scale ratio $R .^{22,24}$ The equilibrium values of these quantities are, respectively, denoted by $\left(\mathcal{P}_{\theta} / \epsilon_{\theta}\right)_{\text {eq }}$ and $R_{\text {eq }}$.
If it is furthermore required that the equation for $\mathcal{E}_{\alpha \alpha} / 2$ provides the right level of production of $\epsilon_{\theta}$, then identification of its production term with the one of the standard modeled $\epsilon_{\theta}$ equation $^{28,30}$ gives

$$
2 \Gamma \mathcal{F}_{\alpha \alpha 2}+C_{A} \overline{u_{2}^{\prime} \theta^{\prime}} \Gamma \frac{\epsilon_{\theta}}{\overline{\theta^{\prime 2}}}=C_{P} \overline{u_{2}^{\prime} \theta^{\prime}} \Gamma \frac{\epsilon_{\theta}}{\overline{\theta^{\prime 2}}},
$$

which can be written as

$$
4\left(\alpha_{1}-\alpha_{3}\right) R_{\mathrm{eq}}^{1 / 2}=C_{P}-C_{A},
$$

where $C_{P}$ is an already determined constant, ${ }^{28,30} C_{P} \simeq 2$. An expression for $\alpha_{1}$ is derived by using Eq. (14) in Eq. (13):

$$
\begin{aligned}
\alpha_{1} R_{\mathrm{eq}}^{1 / 2}= & \frac{C_{P}}{2} \frac{\mathcal{E}_{11} / \mathcal{E}_{22}}{1+2\left(\mathcal{E}_{11} / \mathcal{E}_{22}\right)} \\
& -\frac{\alpha_{\mathcal{R}}-\left(C_{S} R_{\mathrm{eq}}+C_{D}\right)}{6\left(\mathcal{P}_{\theta} / \epsilon_{\theta}\right)_{\mathrm{eq}}} \frac{1-\left(\mathcal{E}_{11} / \mathcal{E}_{22}\right)}{1+2\left(\mathcal{E}_{11} / \mathcal{E}_{22}\right)} .
\end{aligned}
$$

The assumption of weak anisotropy yields an approximation of Eq. (15) namely, that $\alpha_{1} R_{\mathrm{eq}}^{1 / 2}$ should be slightly smaller than $C_{P} / 6$. However, it is known that $R_{\text {eq }}$ is not universal even in the simpler case of an homogeneous scalar field decaying in isotropic turbulence. In the situation of an imposed scalar gradient, the experimental results of Sirivat and Warhaft ${ }^{22}$ show that $R_{\text {eq }}$ is, with some scatter, close to $2 / 3$; the same value can be inferred from the measurements of Mydlarski and Warhaft. ${ }^{8} R_{\text {eq }}$ is three times larger in Gibson and Dakos's experiment. ${ }^{24}$ In passing, the experiment of Sirivat and Warhaft is such that $\left(\mathcal{P}_{\theta} / \epsilon_{\theta}\right)_{\mathrm{eq}} \simeq 1.5$ whereas this ratio is around 2.2 in the case investigated by Gibson and Dakos. The nonuniversality of $R_{\text {eq }}$ precludes estimating reliably $\alpha_{1}$ as a constant. For this reason, it is stated that $\alpha_{1}$ and $\alpha_{3}$ depend on $R$ in such a way that $\alpha_{1} R^{1 / 2}=\alpha_{1}^{\prime}$ and $\alpha_{3} R^{1 / 2}$ $=\alpha_{3}^{\prime}, \alpha_{1}^{\prime}$ and $\alpha_{3}^{\prime}$ being constants.

Antonia and Browne ${ }^{11}$ have measured $\overline{\left(\partial \theta^{\prime} / \partial x\right)\left(\partial u^{\prime} / \partial x\right)}\left(\propto \mathcal{F}_{111}\right)$ and $\overline{\left(\partial \theta^{\prime} / \partial x\right)\left(\partial v^{\prime} / \partial x\right)}\left(\propto \mathcal{F}_{112}\right)$ in a turbulent plane jet. It is straightforward to show that, in this case, using the model expressed by Eq. (12):

$$
\frac{\alpha_{3}}{\alpha_{1}}=\frac{1}{2}\left(1-\frac{b_{2}}{b_{1}} \frac{\mathcal{F}_{111}^{*}}{\mathcal{F}_{112}^{*}}\right)\left(1+\frac{b_{1}^{2}}{b_{2}^{2}}\right)^{-1} .
$$

The ratio of the scalar fluxes, $b_{1} / b_{2} \equiv \overline{u^{\prime} \theta^{\prime}} / \overline{v^{\prime} \theta^{\prime}}$, on a section of the jet can be derived from a study by Antonia ${ }^{38}$ on a heated plane jet. Combining these data with those of Antonia and Browne ${ }^{11}$ on $\mathcal{F}_{111}$ and $\mathcal{F}_{112}, \alpha_{3} / \alpha_{1}$ is indeed found to be approximately constant on a significant portion of a transversal section, ranging from -0.6 at $y / L_{u}=0.3\left(L_{u}\right.$ is the half-maximum velocity width) to -0.4 at $y / L_{u}=0.9$, with a mean value close to -0.5 . This suggests that $\alpha_{3} \simeq-0.5 \alpha_{1}$. The model constants are finally estimated as

$$
\alpha_{3}^{\prime} \simeq-0.5 \alpha_{1}^{\prime}, \quad C_{A}=C_{P}-4\left(\alpha_{1}^{\prime}-\alpha_{3}^{\prime}\right),
$$

and $\alpha_{1}^{\prime}$ slightly smaller than $C_{P} / 6$. 


\section{COMBINED EFFECTS OF SCALAR GRADIENT AND SHEAR}

\section{A. Equations for $\mathcal{E}$ in homogeneous sheared turbulence}

Measurements as well as simulations ${ }^{4,6,39,40}$ have revealed that, in passively heated shear flows, significant anisotropies occur at the level of temperature variance dissipation. This feature has also been emphasized by the experimental study of Tavoularis and Corrsin ${ }^{2,3}$ in homogeneous sheared turbulence. In this situation, the flow direction is $x_{1}$ and the scalar and velocity mean gradients, respectively, $\Gamma$ and $S$, are applied in the $x_{2}$ direction. The mean scalar and velocity fields are thus defined as

$$
\begin{aligned}
& \bar{\theta}=\Gamma x_{2}, \quad \Gamma=\text { constant }, \quad \Gamma>0, \\
& \bar{u}_{i}=S x_{2} \delta_{i 1}, \quad S=\text { constant }, \quad S>0 .
\end{aligned}
$$

Whereas in the case of the pure scalar gradient $\overline{\left(\partial \theta^{\prime} / \partial x_{1}\right)^{2}}$ and $\overline{\left(\partial \theta^{\prime} / \partial x_{3}\right)^{2}}$ are equal and slightly smaller than $\overline{\left(\partial \theta^{\prime} / \partial x_{2}\right)^{2}}{ }^{8}$ Tavoularis and Corrsin's measurements show that $\overline{\left(\partial \theta^{\prime} / \partial x_{2}\right)^{2}} \simeq \overline{\left(\partial \theta^{\prime} / \partial x_{3}\right)^{2}} \simeq 1 . \overline{\left(\partial \theta^{\prime} / \partial x_{1}\right)^{2}}{ }^{3}$ Such a hierarchy of the diagonal terms of $\overline{\left(\partial \theta^{\prime} / \partial x_{i}\right)\left(\partial \theta^{\prime} / \partial x_{j}\right)}$ has also been found in nonhomogeneous shear flows. ${ }^{4,6,11}$

In a homogeneous situation as studied by Tavoularis and Corrsin, ${ }^{2,3}$ the equations for the components of $\mathcal{E}$ derived from Eq. (1) are written as follows:

$$
\begin{aligned}
& \frac{d \mathcal{E}_{11}}{d t}=-4 \Gamma \mathcal{F}_{112}+\mathcal{S}_{11}+\mathcal{D}_{11}, \\
& \frac{d \mathcal{E}_{22}}{d t}=-2 S \mathcal{E}_{21}-4 \Gamma \mathcal{F}_{222}+\mathcal{S}_{22}+\mathcal{D}_{22}, \\
& \frac{d \mathcal{E}_{33}}{d t}=-4 \Gamma \mathcal{F}_{332}+\mathcal{S}_{33}+\mathcal{D}_{33}, \\
& \frac{d \mathcal{E}_{21}}{d t}=-S \mathcal{E}_{11}-2 \Gamma\left(\mathcal{F}_{212}+\mathcal{F}_{122}\right)+\mathcal{S}_{21}+\mathcal{D}_{21} .
\end{aligned}
$$

Analysis of the previous equations reveals complex mechanisms. Mean shear affects $\mathcal{E}_{22}$ through interaction with the off-diagonal component, $\mathcal{E}_{21}$. The off-diagonal component itself results from the interaction of shear with $\mathcal{E}_{11}$. Equations for $\mathcal{E}_{11}$ and $\mathcal{E}_{33}$ are free from direct shear effects.

As in the case of the pure scalar gradient, components $\mathcal{F}_{112}, \mathcal{F}_{222}$, and $\mathcal{F}_{332}$ interact with the latter to produce, respectively, $\mathcal{E}_{11}, \mathcal{E}_{22}$, and $\mathcal{E}_{33}$. Equations for the components of $\mathcal{F}$ (not displayed here) show that interaction of shear with $\mathcal{F}_{112}$ results in the production of $\mathcal{F}_{212}$ and $\mathcal{F}_{122}$. The latter, interacting with the mean scalar gradient, play on $\mathcal{E}_{21} \cdot \mathcal{F}_{222}$ is directly affected by mean shear but $\mathcal{F}_{112}$ and $\mathcal{F}_{332}$ are not. The equations for $\mathcal{F}$ also reveal intricate interactions due to the effect on $\mathcal{F}_{222}$ and $\mathcal{F}_{122}$ of the respective pressure terms $\Pi_{222}$ and $\Pi_{122}$, which, in the present case, include the mean velocity gradient [Eq. (3)].

The direct influence of shear on $\mathcal{E}_{22}$ could explain the predominance of this component in shear flows. However, understanding the reason why, in this type of flow, $\mathcal{E}_{33}$ $\simeq \mathcal{E}_{22}$ is more delicate because $\mathcal{E}_{33}$ does not directly experi- ence the effect of shear. Now, the algebraic model for $\mathcal{F}_{i j k}$ given by Eq. (12) includes an effect of shear through the large-scale anisotropy of the scalar field measured by vector $\boldsymbol{b}$. As a matter of fact, in the case defined by Eqs. (16) and (17), Eq. (12) implies

$$
\frac{\mathcal{F}_{332}^{*}}{\mathcal{F}_{112}^{*}}=1+\frac{b_{1}^{2}}{b_{2}^{2}},
$$

which, in the conditions of Tavoularis and Corrsin's experiment ${ }^{2}\left(b_{1} \simeq 0.43, b_{2} \simeq-0.19\right)$, leads to $\mathcal{F}_{332}^{*} \simeq 6 \mathcal{F}_{112}^{*}$. Production by the mean scalar gradient is thus larger in the $\mathcal{E}_{33}$ equation than in the $\mathcal{E}_{11}$ equation as a consequence of indirect effect of shear on $\mathcal{F}_{i j k}$.

In the following, predictions obtained from the equations for $\mathcal{E}_{11}, \mathcal{E}_{22}, \mathcal{E}_{33}$, and $\mathcal{E}_{21}$ are compared with the experimental data of Tavoularis and Corrsin ${ }^{2,3}$ using the models expressed by Eqs. (10) and (12) for, respectively, $\mathcal{S}_{i j}+\mathcal{D}_{i j}$ and $\mathcal{F}_{i j k}$. In this manner, the effect of shear is taken into account directly in $\mathcal{E}_{21}$ and $\mathcal{E}_{22}$ equations and, indirectly, through its effects on the $\mathcal{F}_{i j k}$ components as well as on $\mathcal{S}_{i j}+\mathcal{D}_{i j}$ via $\mathcal{P}_{i j}^{A}$ [Eq. (10)].

\section{B. Predictions in a homogeneous shear flow \\ 1. Experimental conditions}

Tavoularis and Corrsin ${ }^{2,3}$ have investigated the evolution of a temperature field under influence of a passive, mean transverse gradient $\left(\Gamma=9.5^{\circ} \mathrm{C} \mathrm{m}^{-1}\right)$ in a homogeneous shear flow $\left(S=46.8 \mathrm{~s}^{-1}\right)$. The streamwise, transverse, and spanwise directions are denoted, respectively, by $x_{1}, x_{2}$, and $x_{3}$. The mean centerline velocity is $\bar{u}_{1}\left(x_{2} / h=0.5\right)=U_{c}$ $=12.4 \mathrm{~m} \mathrm{~s}^{-1}$. The height of the shear generator is $h$ $=0.276 \mathrm{~m}$. It can be checked, from the experimental data, that at $x_{1} / h=11, S q^{2} / 2 \epsilon \simeq 6.1$. Homogeneity of the fluctuating velocity and temperature fields as well as of their cross correlations are rather well ensured on a significant transverse portion of the flow. Detailed measurements including the fine-scale structure are provided at $x_{1} / h=7.5, x_{1} / h$ $=9.5$, and $x_{1} / h=11$. At $x_{1} / h=11$, the Reynolds and Péclet numbers based on the longitudinal microscales are, respectively, $\operatorname{Re}_{\lambda_{11}}=266$ and $\mathrm{Pe}_{\lambda_{\theta 1}}=167$.

\section{Model calculations}

Predictions are derived by solving equations for the dynamic field, that is, for $q^{2}$ and $\epsilon$ (the equilibrium Reynolds stress anisotropy tensor being given) as well as for the scalar variance, $\overline{\theta^{\prime 2}}$, the scalar fluxes, $\overline{u_{1}^{\prime} \theta^{\prime}}$ and $\overline{u_{2}^{\prime} \theta^{\prime}}$ and the $\mathcal{E}_{i j}$ components. The initial conditions of the model calculations are the experimental data at $x_{1} / h=7.5$. There is no experimental information about anisotropy of the fine-scale temperature field at this section. However, some tests have revealed that calculation is weakly sensitive to the latter and the results presented in the following have been obtained with isotropic initial conditions. Values of constants $\alpha_{1}^{\prime}, \alpha_{3}^{\prime}$, and $C_{A}$ are $\alpha_{1}^{\prime}=0.3, \alpha_{3}^{\prime}=-0.12, C_{A}=0.12$.

Comparison of predictions with experimental results is displayed in Tables I-III. The overall features of the dynamic and scalar fields are rather well predicted (Table I). In 
TABLE I. Comparison of predicted dynamic and scalar fields with Tavoularis and Corrsin's experimental data (TC81- Ref. 2). Unless specified, dimensional quantities are given in SI units. $q^{2}$ is twice the turbulence kinetic energy; $\epsilon$, the mean dissipation of turbulence kinetic energy; $\overline{\theta^{\prime 2}}$, the variance of scalar fluctuations; $\epsilon_{\theta}$, the mean dissipation of scalar fluctuations energy, $\overline{\theta^{\prime 2}} / 2 ; R$, the scalar-to-velocity time scale ratio, $\overline{\theta^{\prime 2}} \epsilon / \epsilon_{\theta} q^{2}$; $\mathcal{P}_{\theta} / \epsilon_{\theta}$, the production-to-dissipation ratio of scalar variance; $\overline{u_{i}^{\prime} \theta^{\prime}}(i$ $=1,2)$, the longitudinal $(i=1)$ and transversal $(i=2)$ scalar turbulent fluxes; the following notations are used: $u_{i}^{\prime} \equiv{\overline{u_{i}^{\prime 2}}}^{1 / 2}$ and $\theta^{\prime} \equiv{\overline{\theta^{\prime 2}}}^{1 / 2}$.

\begin{tabular}{llc}
\hline \hline & TC81 & Prediction \\
\hline$q^{2}$ & 0.89 & 0.83 \\
$\frac{\epsilon}{\theta^{\prime 2}}$ & 3.4 & 3.2 \\
$\epsilon_{\theta}$ & 0.016 & 0.019 \\
$R$ & $0.18(0.13)^{\mathrm{a}}$ & 0.21 \\
$\frac{\mathcal{P}_{\theta} / \epsilon_{\theta}}{u_{1}^{\prime} \theta^{\prime}} / u_{1}^{\prime} \theta^{\prime}$ & $0.34(0.46)^{\mathrm{a}}$ & 0.36 \\
$u_{2}^{\prime} \theta^{\prime}$ & $1.2(1.7)^{\mathrm{a}}$ & 1.1 \\
$\theta^{\prime}$ & 0.59 & 0.52 \\
\hline
\end{tabular}

${ }^{\text {a The first values correspond to } \epsilon_{\theta} \text { estimated from the imbalance of transport }}$ and production in the scalar variance equation; the values in parentheses correspond to $\epsilon_{\theta}$ computed from the sum of the mean squared temperature derivatives.

addition, as shown in Table II, $\mathcal{E}_{11}$ is found to be the weaker diagonal component of $\mathcal{E}$ and the computed off-diagonal anisotropy, measured by $\mathcal{E}_{21} /\left(\mathcal{E}_{11} \mathcal{E}_{22}\right)^{1 / 2}$, compares well with the corresponding experimental value; prediction of the scalar microscales $\lambda_{\theta_{i}}$ and of $\mathcal{F}_{112}$ (the only measured $\mathcal{F}$ component) is reasonable as well. Nevertheless, the ratio $\mathcal{E}_{33} / \mathcal{E}_{22}$ is underestimated. Closer examination of the results is synthesized in Table III, which displays the components of the anisotropy tensor $\mathcal{A}$ and its second and third invariants. These latter are sensitive to departures in the $\mathcal{E}_{i j}$ components and correct prediction of their values is a difficult test. They are reported here in consideration of the qualitative information they provide. In fact, the results in Table III reveal that although the degree of anisotropy is approximately well predicted, its structure differs from the experimental one. Indeed, the computed values of $\mathcal{A}_{11}$ and $\mathcal{A}_{21}$ are correct but $\mathcal{A}_{22}$ is overestimated and $\mathcal{A}_{33}$, though close to its isotropic value (as in the experiment), is not predicted with the right sign. Consequently, although the weak values of the predicted second and third invariants of $\mathcal{A}$ confirm a moderate anisotropy in agreement with experiment, the computed

TABLE II. Comparison of predicted anisotropy at the level of scalar dissipation with Tavoularis and Corrsin's experimental data (TC81 - Ref. 3). $\mathcal{E}_{i j}=2 D \overline{\left(\partial \theta^{\prime} / \partial x_{i}\right)\left(\partial \theta^{\prime} / \partial x_{j}\right)}(D$ is the molecular diffusivity of the scalar); the $\lambda_{\theta_{i}}$ are the scalar microscales, $\lambda_{\theta_{i}}=\left(4 D \overline{\theta^{\prime 2}} / \mathcal{E}_{i i}\right)^{1 / 2}$ (without summation over $i) ; \mathcal{F}_{112}=D \overline{\left(\partial \theta^{\prime} / \partial x_{1}\right)\left(\partial u_{2}^{\prime} / \partial x_{1}\right)}$.

\begin{tabular}{lcc}
\hline \hline & TC81 & Prediction \\
\hline $\mathcal{E}_{22} / \mathcal{E}_{11}$ & 1.8 & 2.4 \\
$\mathcal{E}_{33} / \mathcal{E}_{11}$ & 1.8 & 1.3 \\
$\mathcal{E}_{21} /\left(\mathcal{E}_{11} \mathcal{E}_{22}\right)^{1 / 2}$ & -0.48 & -0.48 \\
$\lambda_{\theta_{1}}(\mathrm{~mm})$ & 5.0 & 4.6 \\
$\lambda_{\theta_{2}}(\mathrm{~mm})$ & 3.7 & 2.9 \\
$\lambda_{\theta_{3}}(\mathrm{~mm})$ & 3.7 & 4.1 \\
$\mathcal{F}_{112}$ & -0.018 & -0.016 \\
\hline \hline
\end{tabular}

TABLE III. Comparison of the predicted structure of anisotropy at the level of scalar dissipation with Tavoularis and Corrsin's experimental data (TC81 - Ref. 3). Typical data related to the case of the pure scalar gradient (PSG) and corresponding to $\mathcal{E}_{11}=\mathcal{E}_{33}, \mathcal{E}_{22}=1.4 \mathcal{E}_{11}$ are also indicated. $\mathcal{A}_{i j}$ are the components of the anisotropy tensor, $\mathcal{A}_{i j}=\mathcal{E}_{i j} / 2 \epsilon_{\theta}-\delta_{i j} / 3$; II ${ }_{\theta}$ and $\mathrm{III}_{\theta}$ are, respectively, the second and third invariants of $\mathcal{A}$ : $\mathrm{II}_{\theta}=-\mathcal{A}_{\alpha \beta} \mathcal{A}_{\beta \alpha} / 2$, III $=\mathcal{A}_{\alpha \beta} \mathcal{A}_{\beta \gamma} \mathcal{A}_{\gamma \alpha} / 3$.

\begin{tabular}{lcll}
\hline \hline & PSG & \multicolumn{1}{c}{ TC81 } & Prediction \\
\hline $\mathcal{A}_{11}$ & -0.04 & -0.12 & -0.12 \\
$\mathcal{A}_{22}$ & 0.08 & 0.059 & 0.19 \\
$\mathcal{A}_{33}$ & -0.04 & 0.059 & -0.07 \\
$\mathcal{A}_{21}$ & 0 & -0.14 & -0.16 \\
$\mathrm{II}_{\theta}$ & $-4.6 \times 10^{-3}$ & $-3 \times 10^{-2}$ & $-5.3 \times 10^{-2}$ \\
$\mathrm{III}_{\theta}$ & $1.2 \times 10^{-4}$ & $-1.6 \times 10^{-3}$ & $3.4 \times 10^{-3}$ \\
$\left(\mathrm{III}_{\theta} / 2\right)\left(-\mathrm{II}_{\theta} / 3\right)^{-3 / 2}$ & 1 & -0.8 & 0.72 \\
\hline \hline
\end{tabular}

quantity $A_{\theta}=\left(\mathrm{III}_{\theta} / 2\right)\left(-\mathrm{II}_{\theta} / 3\right)^{-3 / 2}$ does not agree with the measured one in that the former corresponds to the right lower part of the anisotropy invariant map ( $\left.-\mathrm{II}_{\theta} \mathrm{vs}^{\mathrm{III}} \mathrm{I}_{\theta}\right)$ (Ref. 6) whereas the latter is close to the left boundary (axisymmetry with $A_{\theta}=-1$ ). Moreover, as already mentioned, measurements and simulations in nonhomogeneous flows ${ }^{4,6,11}$ qualitatively confirm the findings of Tavoularis and Corrsin. ${ }^{3}$ For instance, the numerical simulation of Antonia and $\mathrm{Kim}^{6}$ in a channel flow indeed shows that $\overline{\left(\partial \theta^{\prime} / \partial x_{2}\right)^{2}}$ and $\overline{\left(\partial \theta^{\prime} / \partial x_{3}\right)^{2}}$ are nearly equal and prevail over $\overline{\left(\partial \theta^{\prime} / \partial x_{1}\right)^{2}}$ in the region where $S q^{2} / 2 \epsilon \simeq 6$ and $S /(\epsilon / \nu)^{1 / 2}$ $\simeq 0.1$, which almost corresponds to the conditions of Tavoularis and Corrsin's experiment.

Data relating to the case of forcing by a mean scalar gradient are reported in Table III in order to emphasize the effects resulting from superposing a shear upon the scalar gradient. As can be seen, symmetry between $\mathcal{A}_{11}$ and $\mathcal{A}_{33}$ is broken under the action of shear, since the former is lessened (from -0.04 to -0.12 ) with respect to the zero, isotropic value and the latter is enhanced beyond isotropy (from -0.04 to 0.059 ). Component $\mathcal{A}_{22}$ is slightly reduced (from 0.08 to 0.059 ), which suggests that the increase of $\mathcal{A}_{33}$ occurs mainly at the expense of $\mathcal{A}_{11}$. The way in which the combined actions of scalar gradient and shear are accounted for here apparently does not represent this mechanism exactly. In this regard, the models expressed through $\mathcal{F}_{i j k}$ and the production term $\mathcal{P}_{i j}^{A}$ could be questioned. It is also likely that a relevant effect is absorbed in the terms to be modeled, which requires further investigation to be elucidated. In this regard, recent studies have put forward the key role played by fluctuating vorticity in preferential orientation of the instantaneous scalar gradient. ${ }^{41}$

\section{CONCLUSION}

Examination of the exact equations for $\mathcal{E}_{i j}$ and $\mathcal{F}_{i j k}$ has allowed us to put forward some features of the influence of large-scale forcing felt at the level of scalar dissipation.

In the case of an imposed mean scalar gradient in isotropic turbulence, all three diagonal components of $\mathcal{E}$ experience a direct effect of forcing via interaction of the gradient with components of $\mathcal{F}$. It has been proposed that, in this situation, the observed anisotropy of $\mathcal{E}$ results from addi- 
tional production (felt through the stretching term) due to cliffs moving along the gradient. A model for this mechanism including the large-scale anisotropy of the scalar field and, thereby, possible indirect effect of shear has been devised.

In the situation of combined mean scalar gradient and shear, the picture is more complex. Mean shear selectively promotes some components of $\mathcal{E}$ and $\mathcal{F}$. In addition, smallscale anisotropy of the velocity field induced by shear implies contamination of the small scales of the scalar field; this mechanism is represented by the interaction between the scalar gradient and components of $\boldsymbol{e}$. The influence of shear is also felt through the correlation between scalar gradient and pressure Hessian.

The modeled equations for the components of $\mathcal{E}$ including an algebraic model for $\mathcal{F}_{i j k}$ have been solved in the situation of a mean scalar gradient imposed in a homogeneous sheared turbulence. A mechanism of return to isotropy of $\mathcal{E}$ has been assumed and modeled by a relaxation term the time scale of which is proportional to the scalar dissipation time scale. The latter process would, however, deserve specific investigations. Comparisons of predictions with the experimental data of Tavoularis and Corrsin ${ }^{2,3}$ are moderately satisfying. The overall anisotropy of $\mathcal{E}$ and the hierarchy of its diagonal components are retrieved as well as the off-diagonal anisotropy. Nevertheless, the fine structure described by the anisotropy tensor and its second and third invariants is not predicted exactly. This induces one to think that, although the proposed models include relevant mechanisms, the scenario describing contamination of the scalar field at small scales has to be improved when shear is present.

${ }^{1}$ K. R. Sreenivasan and S. Tavoularis, " On the skewness of the temperature derivative in turbulent flows," J. Fluid Mech. 101, 783 (1980).

${ }^{2}$ S. Tavoularis and S. Corrsin, "Experiments in nearly homogeneous turbulent shear flow with a uniform mean temperature gradient. 1,' J. Fluid Mech. 104, 311 (1981).

${ }^{3}$ S. Tavoularis and S. Corrsin, "Experiments in nearly homogeneous turbulent shear flow with a uniform mean temperature gradient. 2 . The fine structure,' J. Fluid Mech. 104, 349 (1981).

${ }^{4}$ L. V. Krishnamoorthy and R. A. Antonia, "Temperature-dissipation measurements in a turbulent boundary layer," J. Fluid Mech. 176, 265 (1987).

${ }^{5} \mathrm{~K}$. R. Sreenivasan, ' On local isotropy of passive scalars in turbulent shear flows,” Proc. R. Soc. London, Ser. A 434, 165 (1991).

${ }^{6}$ R. A. Antonia and J. Kim, "A numerical study of local isotropy of turbulence," Phys. Fluids 6, 834 (1994).

${ }^{7} \mathrm{C}$. Tong and Z. Warhaft, "On passive scalar derivative statistics in grid turbulence," Phys. Fluids 6, 2165 (1994).

${ }^{8}$ L. Mydlarski and Z. Warhaft, "Passive scalar statistics in high-Péclet number grid turbulence," J. Fluid Mech. 358, 135 (1998).

${ }^{9} \mathrm{C}$. W. Van Atta, "Second-order spectral local isotropy in turbulent scalar fields," J. Fluid Mech. 80, 609 (1977).

${ }^{10}$ J. L. Lumley and B. Khajeh-Nouri, "Computational modeling of turbulent transport," Adv. Geophys. 18, 169 (1974).

${ }^{11}$ R. A. Antonia and L. W. B. Browne, "The destruction of temperature fluctuations in a turbulent plane jet,' J. Fluid Mech. 134, 67 (1983).

${ }^{12} \mathrm{M}$. Tagawa, Y. Nagano, and T. Tsuji, "Turbulence model for the dissipation components of Reynolds stresses," Eighth Symposium on Turbulent Shear Flows (Technical University of Munich, Munich, Germany, 1991).

${ }^{13} \mathrm{M}$. Oberlack, "Non-isotropic dissipation in non-homogenenous turbulence," J. Fluid Mech. 350, 351 (1997).

${ }^{14}$ C. G. Speziale and T. B. Gatski, "Analysis and modeling of anisotropies in the dissipation rate of turbulence," J. Fluid Mech. 344, 155 (1997).
${ }^{15}$ J. L. Lumley, "Computational modeling of turbulent flows," Adv. Appl. Mech. 18, 123 (1978).

${ }^{16} \mathrm{~K}$. Ohkitani and S. Kishiba, "Nonlocal nature of vortex stretching in an inviscid fluid," Phys. Fluids 7, 411 (1995).

${ }^{17}$ K. K. Nomura and G. K. Post, "The structure and dynamics of vorticity and rate of strain in incompressible homogeneous turbulence," J. Fluid Mech. 377, 65 (1998).

${ }^{18} \mathrm{P}$. Vieillefosse, "Internal motion of a small element of fluid in an inviscid flow," Physica A 125, 150 (1984).

${ }^{19}$ B. J. Cantwell, "Exact solution of a restricted Euler equation for the velocity gradient tensor," Phys. Fluids A 4, 782 (1992).

${ }^{20}$ J. Martín, C. Dopazo, and L. Valiño, "Dynamics of velocity gradients invariants in turbulence: Restricted Euler and linear diffusion models," Phys. Fluids 10, 2012 (1998).

${ }^{21}$ S. Corrsin, "Heat transfer in isotropic turbulence," J. Appl. Phys. 23, 113 (1952).

${ }^{22}$ A. Sirivat and Z. Warhaft, "The effect of a passive cross-stream temperature gradient on the evolution of the temperature variance and heat flux in grid turbulence,"' J. Fluid Mech. 128, 323 (1983).

${ }^{23}$ R. Budwig, S. Tavoularis, and S. Corrsin, "Temperature fluctuations and heat flux in grid-generated isotropic turbulence with streamwise and transverse mean-temperature gradient," J. Fluid Mech. 153, 441 (1985).

${ }^{24}$ M. M. Gibson and T. Dakos, "Production of temperature fluctuations in grid turbulence: Wiskind's experiment revisited," Exp. Fluids 16, 146 (1993).

${ }^{25} \mathrm{~S}$. T. Thoroddsen and C. W. Van Atta, "Exponential tails and skewness of density gradient probability density functions in stably stratified turbulence,'’ J. Fluid Mech. 244, 547 (1992).

${ }^{26}$ M. Holzer and E. D. Siggia, "Turbulent mixing of a passive scalar," Phys. Fluids 6, 1820 (1994).

${ }^{27}$ A. Pumir, "A numerical study of a passive scalar in three dimensions in the presence of a mean gradient,"' Phys. Fluids 6, 2118 (1994).

${ }^{28}$ G. R. Newman, B. E. Launder, and J. L. Lumley, "Modeling the behaviour of homogeneous scalar turbulence,'” J. Fluid Mech. 111, 217 (1981).

${ }^{29}$ S. E. Elghobashi and B. E. Launder, "Turbulent time scales and the dissipation rate of temperature variance in the thermal mixing layer," Phys. Fluids 26, 2415 (1983).

${ }^{30}$ T. H. Shih and J. L. Lumley, "Influence of timescale ratio on scalar flux relaxation: modeling Sirivat \& Warhaft's homogeneous passive scalar fluctuations," J. Fluid Mech. 162, 211 (1986).

${ }^{31}$ W. P. Jones and P. Musonge, "Closure of the Reynolds stress and scalar flux equations,"' Phys. Fluids 31, 3589 (1988).

${ }^{32}$ O. Zeman and J. L. Lumley, "Buoyancy effects in entraining turbulent boundary layers: A second-order closure study," in Turbulent Shear Flows 1, edited by F. Durst, B. E. Launder, F. W. Schmidt, and J. H. Whitelaw (Springer, Berlin, 1979).

${ }^{33}$ L. W. B. Browne, R. A. Antonia, and D. A. Shah, "Turbulent energy dissipation in a wake," J. Fluid Mech. 179, 307 (1987).

${ }^{34} \mathrm{~S}$. T. Thoroddsen and C. W. Van Atta, "Experiments on density gradients anisotropies and scalar dissipation of turbulence in a stably stratified fluid,' J. Fluid Mech. 322, 383 (1996).

${ }^{35}$ N. Shikazono and N. Kasagi, "Second-moment closure for turbulent scalar transport at various Prandtl numbers," Int. J. Heat Mass Transf. 39, 2977 (1995).

${ }^{36}$ H. S. Dol, K. Hanjalić, and S. Kenjereš, "A comparative assessment of the second-moment differential and algebraic models in turbulent natural convection,"' Int. J. Heat Fluid Flow 18, 4 (1997).

${ }^{37}$ M. Wörner, Q. Y. Ye, and G. Grötzbach, "Consistent modeling of fluctuating temperature gradient-velocity gradient correlations for natural convection," in Engineering Turbulence Modeling and Experiments 4, edited by W. Rodi and D. Laurence (Elsevier, New York, 1999).

${ }^{38}$ R. A. Antonia, "On a heat transport model for a turbulent plane jet," Int. J. Heat Mass Transf. 28, 1805 (1985).

${ }^{39}$ R. A. Antonia, F. Anselmet, and A. J. Chambers, "Assessment of local isotropy using measurements in a turbulent plane jet," J. Fluid Mech. 163, 365 (1986).

${ }^{40}$ R. A. Antonia and L. W. B. Browne, "Anisotropy of the temperature dissipation in a turbulent wake," J. Fluid Mech. 163, 393 (1986).

${ }^{41}$ P. J. Diamessis and K. K. Nomura, "Interaction of vorticity, rate-of-strain and scalar gradient in stratified homogeneous turbulence," Phys. Fluids 12, $1166(2000)$ 